Niniejsza publikacja jest dostęna na licencji Creative Commons. Uznanie autorstwa-Użycie niekomercyjne-Bez utworów zależnych 3.0 Polska. Pewne prawa zastrzeżone na rzecz autora. Zezwala się na wykorzystanie publikacji zgodnie z licencja - pod warunkiem zachowania niniejszej informacji licencyjnej oraz wskazania autora jako właściciela praw do tekstu. Treść licencji jest dostępna na stronie: http://creativecommons.org/licenses/by-nc-nd/3.0/pl/

Lingwistyka Stosowana 19: 4/2016, 23-31

\author{
Beate BAUMANN
}

Universität Catania

\title{
Die Entdeckung des „Selbstverständlichen in einem neuen Licht““ Interkulturelles Lernen durch Spracharbeit und -reflexion im uni- versitären Kontext
}

\begin{abstract}
:
The discovery of the „obvious in a new light“. Intercultural learning through language work and language reflection

This article starts out from an empirically based research project that was conducted with GFL students at the University of Catania and Italian L2 students at the Freie Universität Berlin. The overall objectives were consolidation and expansion of intercultural competence which should be reached methodically by means of language and cultural work with texts of transcultural literature. Against this background, the cultural-oriented learning processes of students will be analysed and described with the aid of explorative-interpretative qualitative research methods.
\end{abstract}

\section{Einführende Überlegungen}

Dass angesichts der zunehmenden Vernetzung unserer Gesellschaften im Zuge von Globalisierungs- und Migrationstendenzen interkulturelle Kompetenzen als fundamentale Schlüsselqualifikation in nahezu allen Lebensbereichen, ganz besonders aber im beruflichen Kontext, angesehen werden, darüber herrscht weitgehend Konsens. Wie jedoch diese Schlüsselqualifikation im Einzelnen zu definieren ist und auf welche Weise sie vermittelt bzw. angeeignet werden kann, dazu liegen vielfältige Vorschläge und Ansätze vor, die in der Wissenschaftsgemeinschaft weiterhin ausgiebig diskutiert werden, da Interkulturalität und interkulturelles Lernen in der, globalisierten bzw. glokalisierten Welt unserer Tage [...] keine Option mehr" darstellt, sondern „ein regulatives Ideal und normatives Gebot, das seine Wurzeln in realen Bedingungen und praktischen Notwendigkeiten hat.“ (J. Straub 2010: 33. Hervorhebung im Original).

Einen Versuch, dieses Postulat im akademischen Kontext konkret in die Wirklichkeit umzusetzen, stellt das Forschungs- und Begegnungsprojekt der Universität Catania und der Freien Universität Berlin dar, das im Folgenden vorstellt werden soll. Dabei soll vor dem Hintergrund eines mehrdimensionalen Konstrukts interkultureller Kompetenz insbesondere die Dimension der Sprach- und Kulturarbeit im Umgang mit transkulturellen Literaturtexten beleuchtet werden, die einen zentralen 
methodischen Zugang zum Erwerb und Ausbau interkultureller Kompetenzen darstellten.

\section{Zum Projekt}

Ausgangspunkt für die folgenden Überlegungen ist ein empirisch angelegtes internationales Forschungs- und Begegnungsprojekt, das im Studienjahr 2014-2015 mit fortgeschrittenen DaF- bzw. Italienisch-L2-Studierenden (Ausgangsniveau B2 GER) durchgeführt wurde, die die Masterkurse „Deutsche Linguistik und Übersetzung“ (Fremdsprachen- und Literaturstudiengang) an der Universität Catania und „Kompetenzorientierung im Italienischunterricht" (Lehramtsstudiengang) an der Freien Universität Berlin besuchten. Die Zielsetzung des Projektes mit dem Titel Interkulturelles Lernen im internationalen universitären Kontext. Ein Austauschprojekt mit Texten zur deutsch- und italienischsprachigen Migrationsliteratur orientierte sich an der Überzeugung von dem ,unauflöslichen Zusammenhang von Sprache und Denken, von Denken und Kultur und von Kultur und Sprache“ (K. Ehlich 2006: 60). Insbesondere durch ein durch Kooperation bestimmtes Lernszenario sollte die immanente Mehrsprachigkeit und kulturelle Vielschichtigkeit, die die Spezifik dieser literarischen Texte ausmachen, erkundet werden, um eine Konsolidierung bzw. Erweiterung interkultureller Kompetenzen zu fördern.

Die Zusammenarbeit auf didaktischer Ebene stellte das Kernstück des Projektes dar, dessen komplex artikulierter theoretisch-wissenschaftliche Bezugsrahmen in diesem Kontext nicht näher erläutert werden soll (vgl. hierzu B. Baumann 2016). Dennoch soll in diesem Zusammenhang auf einen für dieses Projekt zentralen Aspekt hingewiesen werden, nämlich auf den einer angemessenen Konzeptualisierung dessen, was in diesem spezifischen Kontext interkulturelle Kompetenz ausmacht. Als Grundlage dient das Strukturmodell der Interkulturellen Kommunikativen Kompetenz M. Byrams (1997), das nach wie vor ein wichtiges Bezugsmodell darstellt. Doch schien es angesichts der Arbeit mit und über Sprache in transkulturellen Literaturtexten angemessen und sinnvoll, dieses Grundlagenmodell durch eine kulturwissenschaftliche Perspektive zu erweitern. Zum einen lässt sich hierzu das Konzept der kulturellen Deutungsmuster heranziehen, das insbesondere von C. Altmayer $(2004,2006)$ für den DaF-Bereich adaptiert und weiterentwickelt hat wurde und als richtungsweisend für interkulturelle Verstehens- und Lernprozesse erachtet wird. Auf der anderen Seite erwies sich gerade beim Umgang mit literarischen Texten der von C. Kramsch $(2006,2009,2011)$ entwickelte Ansatz der symbolic competence, der bislang vor allem in der Literatur- und Kulturdidaktik rezipiert wurde, aber auch in der Fremdsprachendidaktik größere Aufmerksamkeit verdienen würde, als ausgesprochen nützlich. Das Konzept der symbolischen Kompetenz richtet die Aufmerksamkeit auf die Komplexität und Ambivalenz sprachlicher Äußerungen, aber auch auf die Bedeutungshaftigkeit der sprachlichen Form selbst, der „form as meaning“ (C. Kramsch 2006: 251).

In Bezug auf die empirische Erforschung der kulturbezogenen Lernprozesse der Studierenden, die durch die Spracharbeit mit den Literaturtexten und der damit einhergehenden Sprachreflexion in Gang gesetzt wurden, wurde umfangreiches Daten- 
material gesammelt und zusammengestellt, das es explorativ-interpretativ zu untersucht gilt. Hierzu zählen beispielsweise Videografien mit sprachbiografischen Interviews, Videokonferenzen der Kursbegegnungen, Unterrichtsbesprechungen, direkte Kursprodukte (Präsentationen der Gruppenarbeiten, Übersetzungen, Hausarbeiten) sowie Materialien, die metalinguistische und metakulturelle Reflexionen der Studierenden enthalten (Evaluationen der Gruppenarbeiten und des Gesamtprojektes, Lerntagebücher). Die Datensätze sollen miteinander trianguliert und durch die Forschungsmethode der qualitativen Inhaltsanalyse (U. Kuckartz 2014, Ph. Mayring 2015) analysiert und ausgewertet werden, um kulturbezogene Lernprozesse nachvollziehbar und rekonstruierbar zu machen und Rückschlüsse auf die Erweiterung interkultureller Kompetenzen zu ziehen.

\section{Sprach- und Kulturarbeit mit transkulturellen Literaturtexten: sprachstilis- tische und translatorische Textarbeit als methodischer Zugang}

Fremdsprachige und in besonderem Maße transkulturelle literarische Texte vermögen die Lernenden zu involvieren und somit ihre Fähigkeit zur Perspektivenübernahme und Perspektivenkoordination zu aktivieren. Aufgrund ihrer identifikatorischen, emotionsbestimmten Dimension sprechen sie die persönliche Erfahrungswelt der Lernenden an, die sich ebenso wie die literarischen Figuren mit der Situation einer fremden Sprache und Kultur konfrontiert sehen. Erwirkt wird dies vor allem durch die „(ver)fremde(te) Sprache” (S. Schiedermair 2014), die zu einem Verfahren zur Darstellung und Inszenierung von kultureller Heterogenität wird. Die Auseinandersetzung auf sprachlich-stilistischer Ebene mit lexikalischen Hybridisierungserscheinungen, die zumeist durch mehrsprachige, sinnlich-körperliche und bildhafte Elemente gekennzeichnet sind, lässt die Vielschichtigkeit, Differenzierungen, Ambivalenzen und Widersprüchlichkeiten nicht nur sprachlicher Äußerungen, sondern kultureller Identitäten sichtbar werden.

Die Konfrontation mit sprachlich-stilistischen Fragenstellungen spielt eine wichtige Rolle auch bei der Übertragung komplexer hybridsprachlicher und -kultureller Erscheinungen in den Kontext der Herkunftskultur der Lernenden, deren neue Muster in der Zielkultur als ungewöhnlich wahrgenommen, aber durchaus auch als Bereicherung akzeptiert werden können (G. L. Schiewer 2011: 66). In der Hinsicht scheint eine Erweiterung des engeren, sprachbezogenen Übersetzungsbegriffes erforderlich hin zu einem Konzept, das die Übersetzung als kulturelle Praxis und Handlungsform begreift. In diesem Zusammenhang ist auch die metaphorische Ausweitung des Übersetzungsbegriffes und das Konzept der „kulturellen Übersetzung“" zu sehen. Diesbezüglich erläutert H. K. Bhabha (2000: 257):

Kultur (...) ist sowohl transnational als auch translational. (...) Die transnationale Dimension kultureller Transformation - Migration, Diaspora, De-platzierung, Neuverortung - lässt den Prozess kultureller Translation zu einer komplexen Form der Signifikation werden. Der natürliche oder naturalisierte, einheitsstiftende Diskurs (...) kann hier wohl kaum als Bezugspunkt dienen. Der große, wenngleich beunruhigende Vorteil dieser Situation besteht darin, dass sie uns ein stärkeres Bewusstsein von der Kultur als Konstruktion und von der Tradition als Erfindung verschafft. 
Die kulturelle Übersetzung zielt auf die „Übertragung von Vorstellungsinhalten, Werten, Denkmustern, Verhaltensmustern und Praktiken eines kulturellen Kontextes in einen anderen“" (B. Wagner 2012: 1) ab, ohne dabei sprachlich-textuelle, d.h. auch literarische Repräsentationsdimensionen auszuschließen. Ebenso dient sie der „Konzeptualisierung des Prozesses von Bedeutungskonstruktionen, die freilich gerade im Migrationskontext besonders virulent erscheinen“. Damit kann sie auch als „,mentaler Prozess" (G. Vorderobermeier / M. Wolf 2008: 13) begriffen werden, der kulturelle Unterschiede bewusst werden lässt mit der Folge, gemeinhin bekannte und verbreitete Deutungs- und Handlungsmuster in Frage zu stellen. Und genau auf diese Weise werden interkulturelle Verstehens- und Lernprozesse in Gang gesetzt, die für die Erweiterung und Vertiefung interkultureller Kompetenzen förderlich sind.

\section{Lavinia und die Entdeckung des „Selbstverständlichen in einem neuen Licht“1}

Vor dem Hintergrund der oben dargelegten theoretischen Überlegungen wurde das in der didaktischen Phase des Projektes zusammengestellte Datenmaterial gesichtet und aufbereitet. Auch wenn eine umfassende Auswertung noch nicht erfolgt ist und noch keine endgültigen Ergebnisse vorliegen, lassen die Daten dennoch Rückschlüsse auf Zwischenergebnisse zu, die auf konstruktive interkulturelle Verstehens- und Lernprozesse hindeuten.

Hierzu soll als Beispiel ein Einblick in den kulturbezogenen Lernprozess der Studentin Lavinia gegeben werden. Lavinia, 24 Jahre alt, stammt aus einer sizilianischen Familie, durch die sie ihre ersten interkulturellen Erfahrungen sammeln konnte, sowohl innerhalb Italiens - ihre Eltern haben einige Zeit in der Region Venetien gelebt und den beiden Töchtern die kulturellen Unterschiede zwischen Nord- und Süditalien vermitteln können - als auch auf zahlreichen Reisen im europäischen und außereuropäischen Ausland. Lavinias Interesse für Fremdsprachen ist auf diese Weise entstanden. ${ }^{2}$

Im Folgenden werden ihre metalinguistischen und -kulturellen Reflexionen in Bezug auf einige Textstellen der Erzählung Mutterzunge von Emine Sevgi Özdamar vorgestellt, die aus ihrem Lerntagebuch stammen. Hier hält sie Inhalte unterschiedlichster Art fest, wie beispielsweise die Beschreibung der Kursinhalte und des Kursgeschehens, Reflexionen und Kommentare zu methodisch-didaktischen Vorgehensweisen, zu den im Unterricht geführten Diskussionen, Gruppenarbeiten (kursintern und mit der Berliner Partnerin) und Videokonferenzen. Häufig vernetzt sie ihre Überlegungen mit Wissen aus anderen Fachbereichen, geht also interdisziplinär vor. Dies alles geschieht in einem höchst individuell-persönlichen Stil, nahezu ein innerer Monolog

\footnotetext{
${ }^{1}$ Zur Anonymisierung der Daten wurden die Namen der Studierenden geändert. Das Originalzitat des Lerntagebucheintrags vom 15.12.2015 lautet: „Riesce a farci pensare, a far vedere quelle che noi consideriamo ovvietà sotto un'altra luce e da un'altra prospettiva.“ (Hervorhebung BB). Die Übersetzungen der italienischen Texte von BB.

${ }^{2}$ Diese Informationen wurden in einem sprachbiographischen Interview mit der Studierenden erhoben.
} 
oder besser ein Dialog mit sich selbst, wobei sie zahlreiche Fragen aufwirft, auf die sie eine Antwort zu geben versucht, die aber z.T. offen bleiben.

So setzt sich Lavinia mit den Besonderheiten des Sprachstils der Erzählung auseinander und denkt über deren Übersetzung und über die damit verbundenen kulturellen Phänomene und Deutungsmuster nach, beispielsweise in Bezug auf die kreative Wortschöpfung „Mutterzunge“ und die Schwierigkeiten, die ihr die Übersetzung in die italienische Sprache bereitet:

Das Erste, was ich gedacht habe, war, dass es sich um ein Unternehmen handelt, bei dem man den Verstand verliert, für einen italienischen Übersetzer, den Unterschied zwischen Zunge und Sprache deutlich zu machen, wenn wir im Italienischen für beides „lingua“ benutzen. Und so ist die Fußnote der italienischen Übersetzung, auf die ich heimlich einen Blick geworfen habe, unvermeidbar gewesen, um den Unterschied gut zu erklären. Wie frustrierend muss es wohl für einen Übersetzer sein, mit lauter Fußnoten zu übersetzen! Aber wie soll das sonst funktionieren? Wie kann man dasselbe kulturelle Konzept in einer anderen Sprache und also auch in einer anderen Kultur wiedergeben? Das ist für mich ein regelrechtes Paradox, das, wenn es mich einerseits „,um den Verstand bringt“, mich anderseits fasziniert. ${ }^{3}$ (Hervorhebung BB)

Diesem Auszug lassen sich zwei Ebenen entnehmen, auf denen Lavinia ihre mentalen Repräsentationen ansiedelt: zum einen eine metakognitive Ebene, auf der sie sich der Komplexität und Ambiguität der (fremd)sprachlichen Zeichen bewusst wird (Unterschied zwischen Sprache und Zunge, lingua, Fußnote, dasselbe kulturelle Konzept in einer anderen Sprache und also auch in einer anderen Kultur), für die es keine direkten Entsprechungen in den jeweiligen Sprachen gibt und die nur durch ein erläuterndes Vorgehen in Form von Fußnoten in der Herkunftssprache und kultur wiedergegeben werden können. Auf der anderen Seite fallen die emotional geprägten Äußerungen auf, die diese Problematik und die für sie unbefriedigende Lösung der Fußnoten bei ihr auslösen (den Verstand verlieren, frustierend, lauter ${ }^{4}$ Fußnoten, Paradox, faszinieren).

\footnotetext{
${ }^{3}$ La prima cosa che ho pensato è stata che deve essere una impresa da perderci il senno, per un traduttore italiano, cercare di rendere la differenza tra Zunge e Sprache, quando noi in italiano usiamo per entrambe le cose "lingua". Ed infatti nella traduzione italiana che ho sbirciato, la nota a piè pagina per spiegare bene la differenza è stata inevitabile. Quanto deve essere frustrante per un traduttore dover tradurre a furia di note a piè pagina! Ma come si fa? Come si rende lo stesso concetto culturale attraverso una lingua diversa e dunque una cultura diversa? Questi sono per me dei veri e propri paradossi, che se da un lato mi fanno "perdere il senno", dall'altro mi affascinano. (Original Lerntagebucheintrag 03./05.12.2014) (Hervorhebung BB).

${ }^{4}$ Ein ähnliches Problem ergibt sich bei der Übersetzung des von der Studierenden verwendeten Begriffes ,a furia di“. Die deutlich emotional geprägte Konnotation des Lexems „furia“ (Wut, Raserei) dieser Wortverbindung mit instrumenteller Funktion kann im Deutschen leider nicht entsprechend wiedergegeben werden.
} 
Zudem setzen die sprachliche Komplexität und Ambiguität dieses Kompositums bei Lavinia einen Reflexionsprozess in Gang, der auf die sprachbiografischen Erfahrungen der Autorin ausgeweitet wird:

Mich hat die Tatsache beeindruckt, dass die Schriftstellerin sagt, sie habe ihre Muttersprache verloren, und vor allem die Implikationen, die dies mit sich führt, worüber wir auch in der Klasse gesprochen haben. „Mutterzunge“. Muttersprache oder die Sprache ihrer Mutter?

Beeindruckt hat mich auch, dass die Autorin, nachdem sie in Berlin gewesen ist und ihre Muttersprache verloren hat, diese dann als eine Art Fremdsprache definiert hat, die sie gut gelernt hatte. Geschieht das wirklich so? Vergisst man letztendlich, wenn man in einem anderen kulturellen und sprachlichen Kontext lebt, seine eigene Herkunft? In Wirklichkeit aber benutzte die Autorin, wenn ich mich nicht täusche, im Text auch einige andere Begriffe in türkischer Sprache. Dann hat sie sie also doch nicht vergessen, diese ihre Sprache. ${ }^{5}$ (Hervorhebung BB)

Sie vollzieht hier einen zweifachen Perspektivenwechsel, indem sie auf der einen Seite die Sichtweise der Ich-Erzählerin einnimmt, zum anderen verallgemeinernd die Konsequenzen daraus, insbesondere in Bezug die eigene sprachliche und kulturelle Identität, ableitet. Konsequenzen, die das Leben in einem neuen kulturellen und sprachlichen Kontext für den Rückblick auf die Herkunftskultur und -sprache hat:

Nachdem man die Erfahrung der Unabhängigkeit vom heimischen Herd gemacht hat, ändert sich sicherlich etwas im Inneren auf unabwendbare Weise. Und wenn man zurückkehrt, dann sieht man die Dinge mit anderen Augen, vielleicht mit kritischeren. Vielleicht ist das die Message, die Özdamar mitteilen wollte? ${ }^{6}$ (Hervorhebung $\mathrm{BB})$

An diesen wenigen Äußerungen lässt sich nachvollziehen, dass das Hinterfragen stilistisch markierter lexikalischer Elemente zu einem Zwischen-den Zeilen-Lesen und zur Rekonstruktion des impliziten Hintergrundwissens und damit kultureller Deutungsmuster führt. Hierbei wird bewusst ein Perspektivenwechsel (Und wenn man zurückkehrt, dann sieht man die Dinge mit anderen Augen, vielleicht mit kritischeren) vollzogen, der über den literarischen Kontext hinausgeht. Dieser veränderte Blickwinkel wird zunächst auf das gesellschaftliche Umfeld im weiteren Sinne

\footnotetext{
${ }^{5} \mathrm{Mi}$ ha colpito il fatto che la scrittrice dice di aver perso la sua lingua madre, e soprattutto le implicazioni che questo comporta, di cui abbiamo anche parlato in classe. "Mutterzunge". La lingua madre, o la lingua di sua madre? (Original Lerntagebucheintrag 03./05.12.2014) [...] E mi ha colpito anche che l'autrice dopo essere stata a Berlino e aver perso la sua lingua madre, l'abbia poi definita come una specie di lingua straniera che aveva imparato bene. Succede davvero così? Quando si vive in un altro contesto culturale e linguistico, si finisce davvero col dimenticare le proprie origini? In realtà però, se non sbaglio, l'autrice usava anche diversi termini in lingua turca nel testo. Dunque non l'ha proprio dimenticata, questa sua lingua. (Original Lerntagebucheintrag 03./05.12.2014) (Hervorhebung BB).

${ }^{6}$ Sicuramente dopo aver fatto un'esperienza di indipendenza dal proprio focolare domestico, qualcosa dentro cambia inesorabilmente. E quando si torna, si vedono le cose con occhi diversi, forse più critici. Forse è questo il messaggio che la Özdamar voleva comunicare? (Original Lerntagebucheintrag 03./05.12.2014) (Hervorhebung BB).
} 
gerichtet und erfasst auch die persönliche Sphäre, wie folgende Überlegungen in Bezug auf den eigenen Lernprozess deutlich machen:

[...] Ich habe gelernt, die anderen mit anderen Augen zu sehen; jede Person, der ich begegne nicht als Träger einer einzigen, sondern mehrerer Identitäten zu betrachten, Identitäten, die aus mehreren Kulturen, mehreren Sprachen und mehreren Geschichten zusammengesetzt sind. Und deshalb glaube ich sagen zu können, dass ich angefangen habe, auch mich mit anderen Augen zu sehen: die Offenheit und der Kontakt mit anderen Wirklichkeiten formen tagtäglich meine Persönlichkeit und machen auch mich in gewisser Weise zu einem Teil der transkulturellen Welt. ${ }^{7}$ (Hervorhebung BB)

Als Ergebnis ihres Lernprozesses, der durch die Reflexion über sprachstilistische und kulturelle Phänomene geprägt ist, nennt Lavinia explizit die Fähigkeit des Mitanderen-Augen-Sehens, das einen offenen Blick auf andere Menschen und sich selbst, ihre und die eigene kulturelle Vielfalt und Heterogenität zur Folge hat. Alterität wahrzunehmen, sie aus einer neuen Perspektive zu betrachten und die Komplexität der eigenen Identität zu begreifen sind deutliche Hinweise dafür, dass die konkrete Projekterfahrung mit der ,authentische[n] und fiktionale[n] Fremde“ (C. Badstübner-Kizik 2014: 79) ein neues kulturelles Verhaltenspotential aktivieren konnte und nicht zuletzt Ausdruck Symbolischer Kompetenz ist. So spricht die Entdeckung des „Selbstverständlichen in einem neuen Licht“ für einen Lernprozess, der auch im Hinblick einer Konsolidierung und Erweiterung der interkulturellen Kompetenz als erfolgreich zu werten ist.

\section{Bibliographie}

Altmayer, C. (2004), Kultur als Hypertext. München.

Altmayer, C. (2006), Kulturelle Deutungsmuster als Lerngegenstand. Zur kulturwissenschaftlichen Transformation der, Landeskunde'. In: Fremdsprachen lehren und lernen 35, 44-59.

Badstübner-Kizik, C. (2014), Sprache - Literatur - Kultur? Zu den Chancen eines didaktischen Brückenschlages. In: C. Altmayer/ M. Dobstadt/ R. Riedner/ C. Schier (Hrsg.), Literatur in Deutsch als Fremdsprache und internationaler Germanistik. Konzepte, Themen, Forschungsperspektiven. Tübingen, 75-85.

Baumann, B. (2015), Multi, inter oder trans? Zum Wandel des Kulturkonzeptes in den heutigen Gesellschaften und den Auswirkungen auf den Bereich Deutsch als

\footnotetext{
${ }^{7}$ [...] Ho imparato a vedere gli altri con occhi diversi. A considerare ogni persona che mi passa davanti non come portatrice di una sola ma di più identità, composte da più culture, più linguaggi e più storie. $\mathrm{E}$ dunque credo di poter dire che conseguentemente ho cominciato a vedere anche me stessa con occhi diversi: l'apertura ed il contatto con altre realtà plasmano ogni giorno la mia personalità, e rendono anche me, in un certo modo, parte del mondo transculturale. (Original Fragebogen Endevaluation 04.02.2015) (Hervorhebung $\mathrm{BB})$.
} 
Fremdsprache. In: M. Bürger-Koftis/ D. Winkler/ S. Vlasta (Hrsg.), Mehrsprachigkeit und Mehrkulturalität im österreichischen Kontext. Wien (im Druck).

Baumann, B. (2016), Kulturbezogenes Lernen im internationalen universitären Kontext. Ein Austauschprojekt mit Texten zur deutsch- und italienischsprachigen transkulturellen Literatur. In: A. Balfanz/ B. Chołuj (Hrsg.), Interkulturalität und Wissensvermittlung an Hochschulen - didaktischer Umgang mit Differenzen. Frankfurt (Oder)/ Słubice.

Bhabha, H. K. (2000), Die Verortung der Kultur. Tübingen.

Byram, M. (1997), Teaching and Assessing Intercultural Communicative Competence. Clevedon Hall.

Ehlich, K. (2006), Die Vertreibung der Kultur aus der Sprache. 13 kurze Reflexionen. In: Zeitschrift für Germanistische Linguistik (Themenheft: Linguistik und Kulturanalyse), 34/ 1-2, 50-63.

Esselborn, K. (2009), Neue Zugänge zur inter/transkulturellen deutschsprachigen Literatur. In: H. Schmitz (Hrsg.), Von der nationalen zur internationalen Literatur. Transkulturelle deutschsprachige Literatur und Kultur im Zeitalter globaler Migration. Amsterdam/ New York, 43-58.

Kramsch, C. (2006), From Communicative Competence to Symbolic Competence. In: The Modern Language Journal, 90/ 2, 249-252.

Kramsch, C. (2009), Discourse, the simbolic dimension of Intercultural Competence. In: A. Hu/ M. Byram (Hrsg.), Interkulturelle Kompetenz und fremdsprachliches Lernen. Modelle, Empirie, Evaluation / Intercultural competence and foreign language learning: models, empiricism, assessment. Tübingen, 107121.

Kramsch, C. (2011), Symbolische Kompetenz durch literarische Texte. In: Fremdsprache Deutsch, 44, 35-40.

Kuckartz, U. (2014), Qualitative Inhaltsanalyse. Methoden, Praxis, Computerunterstützung. (2., durchgesehene Auflage). Weinheim/ Basel.

Mayring, Ph. (2015), Qualitative Inhaltsanalyse. Grundlagen und Techniken. (12. überarbeitete Auflage.) Weinheim.

Özdamar, E. S. (2006), Mutterzunge. Erzählungen. Hamburg.

Schiedermair, S. (2014), Deutsch als (ver)fremd(et)e Sprache. Literarische Verfremdung als Kategorie im Fach Deutsch als Fremdsprache. In: C. Altmayer/ M. Dobstadt/ R. Riedner/ C. Schier (Hrsg.), Literatur in Deutsch als Fremdsprache und Internationaler Germanistik. Konzepte, Themen, Forschungsperspektiven. Tübingen, 131-140.

Schiewer, G. L. (2011), Von der Literatursprache zu Bücher(n), die ihren Lesern Tore öffnen. Perspektiven der interkulturellen Literaturwissenschaft an der Schnittstelle von Translationswissenschaft und Wissenssoziologie. In: M. Ewert/ R. Riedner/ S. Schiedermair (Hrsg.), Deutsch als Fremdsprache und Literaturwissenschaft. Zugriffe, Themenfelder und Perspektiven. München, 60-78.

Straub, J. (2010), Lerntheoretische Grundlagen. In: A. Weidemann/ J. Straub/ S. Nothnagel (Hrsg.), Wie lehrt man interkulturelle Kompetenz? Theorien, Methoden und Praxis in der Hochschulausbildung. Ein Handbuch. Bielefeld, 31-98. 
Vorderobermeier, G./ Wolf, M. (2008), Einleitung: Bleiben, um zu gehen? Zum Prozesscharakter von Übersetzen und Migration. In: G. Vorderobermeier/ M. Wolf (Hrsg.), Meine Sprache grenzt mich ab... Transkulturalität und kulturelle Übersetzung im Kontext von Migration. Wien, 7-18.

Wagner, B. (2009), Kulturelle Übersetzung. Erkundungen über ein wanderndes Konzept. In: Kakanien revisited. (www.kakanien.ac.at/beitr/postcol/Bwagner2.pdf; Zugriff 28.11.2016).

Welsch, W. (2010), Was ist eigentlich Transkulturalität?. In: L. Darowska/ C. Machold (Hrsg.), Hochschule als transkultureller Raum? Beiträge zu Kultur, Bildung und Differenz. Bielefeld, 39-66. 